\title{
Determining transport parameters for unsaturated porous media in flow-tank experiments using image analysis
}

\author{
K. Inoue ${ }^{1}$, N. Setsune ${ }^{2}$, F. Suzuki ${ }^{2} \&$ T. Tanaka ${ }^{1}$ \\ ${ }^{1}$ Faculty of Agriculture, Kobe University, Japan \\ ${ }^{2}$ Graduate School of Science and Technology, Kobe University, Japan
}

\begin{abstract}
Tracer experiments are carried out in water flow tank with $80 \mathrm{~cm}$ height, $80 \mathrm{~cm}$ length and $20 \mathrm{~cm}$ width, which is filled homogeneously with two different types of sand materials. Brilliant Blue FCF, which makes the flow paths visible, is applied as a point source at the surface of the flow field. Dispersivity and dispersion coefficient are estimated using a time series of images of tracer transition. Experimental results show a linear dependence of dispersion coefficients on tracer velocity at a certain velocity and exhibits non-linearity in the range of higher water content. While values of dispersivity show an independency of rainfall intensity, dispersivity in unsaturated flow region is approximately one order larger than that in saturated flow region, indicating that transport parameters depend strongly on the water content where solute passes through.

Keywords: tracer experiment, unsaturated, parameter estimation, dispersion, image analysis, dye.
\end{abstract}

\section{Introduction}

An understanding of solute transport mechanism through not only saturated but unsaturated zones under various hydraulic conditions is important in environmental protection and agriculture. Fast transport of fertilizers and other agrochemicals into subsurface or groundwater systems has been recognized as a serious threat due to high concentration of solutes. In order to understand and express the transport behavior of contaminants in a subsurface, the transport parameters such as longitudinal and transverse dispersivities are some of the key factors that play an important role in spreading the contaminants. 
Recently, a few of the dye tracer experiments combined with image analysis techniques, which are relatively new approaches, have been conducted to quantify the behavior of dye tracers. Application of image analysis has demonstrated that a time series of digitized images reflecting the movement of dye tracer could be successfully used to monitor solute transport in porous media as well as to estimate transport parameters such as dispersion coefficient, dispersivity (Forrer et al. [1]; Inoue et al. [2]) and retardation factor (Flury and Flühler [3]).

There are many aspects of solute transport and dispersion in unsaturated porous media that still poorly understood. The objectives of this study are to determine dispersivity and dispersion coefficient using image analysis and to gain insight into the nature of dispersion phenomena in unsaturated porous media. A new laboratory flow-tank experiment apparatus designed to study this issue is described.

\section{Materials and methods}

\subsection{Materials}

Dye tracing is widely used to characterize water flow and solute transport behavior in porous media. In the experiments, Brilliant Blue FCF is used as a dye tracer which differentiates the tracer evolution visually from the ambient pore water. Although the specific gravity of dye tracer is 1.0001 measured using the specific gravity meter and the initial concentration of tracer is determined to be low enough to avoid density-induced flow effects, there is no denying that the effect of gravity on solute transport. This dye is chosen based on good contrast with soil for visualizing transport patterns of tracer plume and low toxicity implied by its food dye designation (Flury and Flühler [4]).

In order to investigate the influence of average grain size of sand comprising flow field on solute transport phenomena, two types of silica sand are used. According to the difference of the mean particle size, these materials are named Sand A and B in descending order of its value. These materials are completely washed and saturated before packing to remove organic chemicals attached to the particle surface. In the process of creation of homogeneous flow field formation, flow tank is filled with water and sand of interest from bottom to top in $10 \mathrm{~cm}$ layers to achieve uniform sand packing, resulting in $59 \mathrm{~cm}$ depth of the flow field. Before the conduction of tracer experiments, the water characteristic curves for the drying process are measured and are expressed using van Genuchten's model (van Genuchten [5]), as shown in Figure 1. In Table 1, in addition to VG parameters, properties of sand materials are listed.

\subsection{Displacement experiment}

Vertical and lateral miscible displacement experiments under a two-dimensional condition are conducted to understand the dispersion process in an unsaturated porous medium. Displacement experiments are carried out in a two-dimensional and vertically placed water tank with the dimensions of $80 \mathrm{~cm}$ height $\times 80 \mathrm{~cm}$ 


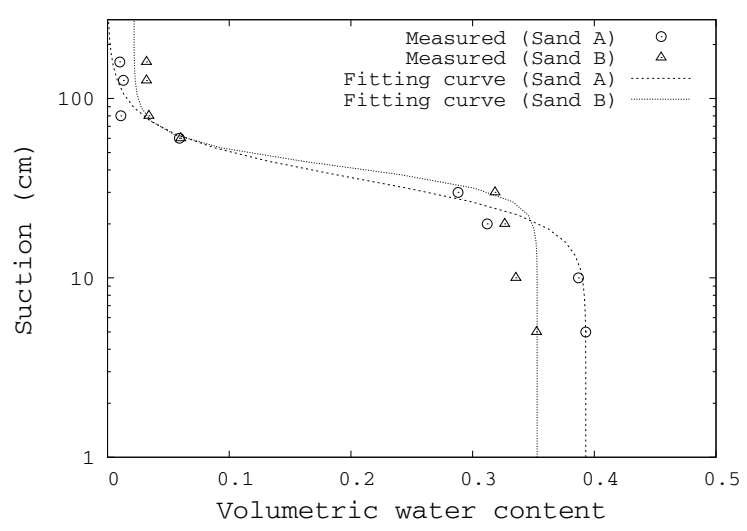

Figure 1: Water characteristic curves for both sand materials.

Table 1: Properties of sand materials used in displacement experiments.

\begin{tabular}{l|cc}
\hline & Sand A & Sand B \\
\hline Particle density $\left(\mathrm{g} / \mathrm{cm}^{3}\right)$ & 2.68 & 2.66 \\
Mean grain size $(\mathrm{cm})$ & 0.085 & 0.050 \\
Uniformity coefficient $(-)$ & 1.80 & 1.25 \\
Hydraulic conductivity at saturation $(\mathrm{cm} / \mathrm{s})$ & 0.751 & 0.268 \\
Volumetric water content at saturation $(-)$ & 0.392 & 0.353 \\
Residual water content $(-)$ & 0.0 & 0.02 \\
VG parameter $n(-)$ & 3.78 & 6.09 \\
VG parameter $\alpha(1 / \mathrm{cm})$ & 0.0311 & 0.0247 \\
\hline
\end{tabular}

length $\times 20 \mathrm{~cm}$ width. The water flow tank is constructed of steel wall and glass plate which enclose a space of a flow field. While the top is open, the water flow tank is closed on three sides by steel walls. The flow tank consists of three distinct sections: two solute transport chambers referred to as Plot 1 and 2 shown in Figure 2 and a tensiometer chamber. Constant head reservoirs are connected to both sides of the tank and are used to keep approximately $55 \mathrm{~cm}$ of the elevation of the water table below the soil surface during the experiments. Schematic diagram of experimental apparatus is shown in Figure 2. The transparent glass plate allows for the observation of migrating dye tracer and the injection of a tracer, while, in order to measure the piezometric head, the stainless steel at the backside of the water tank permits the introduction of 10 piezometric water pressure measurement ports to which standpipes and manometers are connected. 


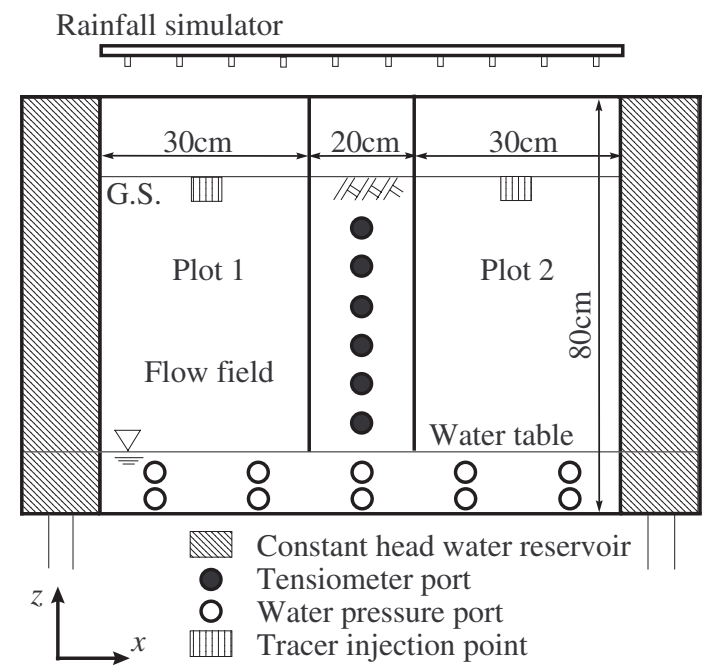

Figure 2: Schematic diagram of the experimental apparatus.

During sand packing, tensiometers are installed at depths of 5, 10, 25, 40, and $55 \mathrm{~cm}$ from the surface and tensions are measured with a hand-held pressure transducer with digital readout. Internal drainage using constant head reservoirs allows for one day to create a saturated and unsaturated flow field after the flow tank is filled with water and sand of concern. A geomembrane with $1 \mathrm{~cm}$ mesh is placed on the soil surface to minimize any disturbance that repeated drops of water might have on the soil surface.

Rainfall applicator of inside dimension of 80 by 40 by $5 \mathrm{~cm}$ is constructed from a $0.5-\mathrm{cm}$ sheet of acrylic plastic. After steady state condition is established, water is applied from 50 needles $0.15-\mathrm{mm}$ i.d., place $50 \mathrm{~cm}$ above the soil surface. Three application rates, $1.29 \times 10^{-2} \mathrm{C} 1.90 \times 10^{-2} \mathrm{C}$ and $2.54 \times 10^{-2} \mathrm{~cm} / \mathrm{s}$, are applied for 60 seconds. Subsequently, $100 \mathrm{~cm}^{3}$ of dye tracers are injected instantaneously at the soil surface for both chambers using four needles to simulate the impulse source having cross-sectional area of approximately $3 \times 3 \mathrm{~cm}^{2}$. These four needles are arranged along the tank width, providing a two-dimensional transport condition. The profiles of tracer evolution are periodically recorded using a digital camera, are then processed and analyzed by image analysis.

\subsection{Image analysis}

In the current context, image analysis refers to the use of variations in brightness or intensity within a digital image as a means of identifying dye tracer evolution of concern. The fundamental assumption is that dye tracer outlines have similar brightness, or tracer concentration. The basic image analysis process included the following steps will be elaborated on: (1) direct digital capture; (2) conversion of 

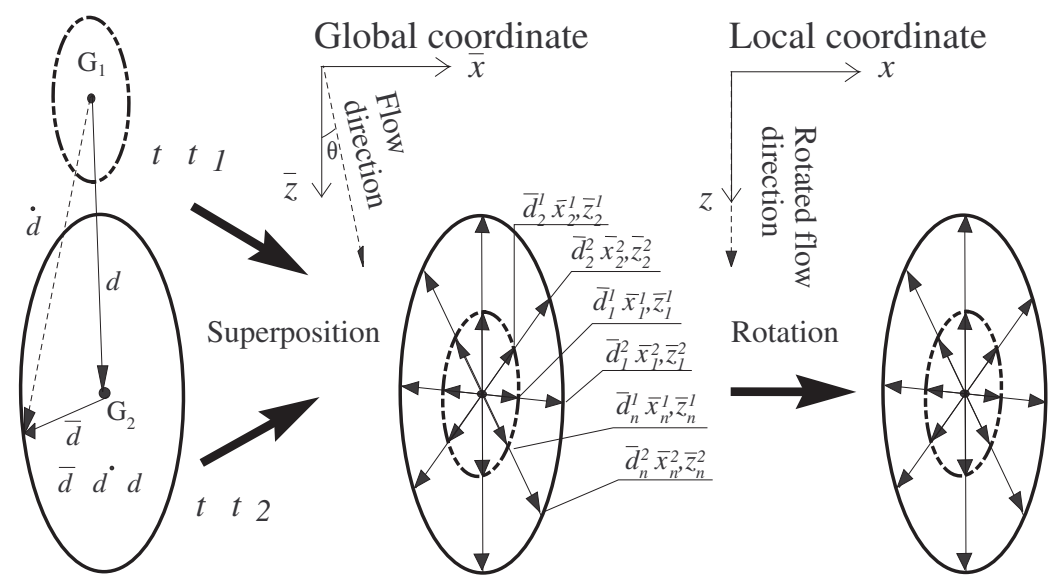

Figure 3: Image analysis process for estimating dispersion coefficient.

the three spectral densities in the red $(R)$, green $(G)$, and blue (B) to binary values (0 and 1); (3) hue discrimination of tracer areas for all pixels in the image at a particular time; (4) extraction of the outlines of tracers; and (5) transformation of the pixel coordinate composing the outlines into real coordinate;

The relation between the dispersion coefficients and the variance of plume is given by the following equation (Bear [6]):

$$
D_{\ell}=\frac{1}{2} \frac{d \sigma_{\ell}^{2}}{d t}
$$

where $\sigma_{\ell}^{2}$ is the longitudinal variance of the plume, $D_{\ell}$ is the dispersion coefficient, and $t$ is time. Assuming that solute particles exist at point $G_{1}$ at $t=t_{1}$ described in Figure 3 and the area of $G_{1}$ is relatively small. Then, the particles move along a mean flow direction. The dispersion coefficient tensor can be estimated by expanding the one-dimensional relationship of eqn (1) to three-dimensional relationship. As shown in Figure 3, the tracer in the porous media at $t=t_{1}$ spreads with movement and its area becomes larger at $t=t_{2}$ than that at $t=t_{1}$. The mean displacement vector of plume during $\Delta t\left(=t_{2}-t_{1}\right)$ can be represented as $\dot{d}$, which corresponds to the vector between centroids of the plume, $G_{1}$ and $G_{2}$. $\bar{x}$ and $\bar{z}$ denote the global coordinates and $x$ and $z$ show the local coordinates. It is assumed that $x$ is the direction from $G_{1}$ to $G_{2}$ and $z$ is perpendicular to $x$. $\theta$ is the angle between $x$ and $\bar{x}$. In order to estimate the dispersion coefficients, digital images are analyzed in the following way: (1) tracer outlines at $t=t_{1}$ and $t=t_{2}$ are extracted and applied to compute the plume centroids $G_{1}$ and $G_{2}$, (2) tracer velocity $\left|V_{d y e}\right|$ is determined from the time $\Delta t$ and the distance between $G_{1}$ and $G_{2}$, (3) vectors $\bar{d}_{n}^{i}\left(\bar{x}_{n}^{i}, \bar{z}_{n}^{i}\right)$ from the centroids of spread are calculated for $n$ directions, where superscript denotes time and subscript shows the number of directions, (4) 
the dispersion coefficient tensor related to tracer movement during $\Delta t$ is estimated based on the following equation (Bear [6]).

$$
D_{i j}=\left(\begin{array}{ll}
D_{\bar{x} \bar{x}} & D_{\bar{x} \bar{z}} \\
D_{\bar{z} \bar{x}} & D_{\bar{z} \bar{z}}
\end{array}\right)=\left(\begin{array}{cc}
\frac{1}{n} \sum_{m=1}^{n} \frac{\bar{x}_{m}^{2}}{2 \Delta t} & \frac{1}{n} \sum_{m=1}^{n} \frac{\bar{x}_{m} \bar{z}_{m}}{2 \Delta t} \\
\frac{1}{n} \sum_{m=1}^{n} \frac{\bar{z}_{m} \bar{x}_{m}}{2 \Delta t} & \frac{1}{n} \sum_{m=1}^{n} \frac{\bar{z}_{m}^{2}}{2 \Delta t}
\end{array}\right)
$$

where $D_{i j}$ is the dispersion coefficient tensor represented in global coordinate. The dispersion coefficient tensor in local coordinate is obtained by rotation.

$$
\left(\begin{array}{ll}
D_{x x} & D_{x z} \\
D_{z x} & D_{z z}
\end{array}\right)=R D_{i j} R^{T}, \quad R=\left(\begin{array}{cc}
\cos \theta & \sin \theta \\
-\sin \theta & \cos \theta
\end{array}\right)
$$

where $T$ means the transpose matrix and $D_{x x}$ and $D_{z z}$ denote longitudinal and transverse dispersion coefficients, respectively. Dispersivities are estimated from dye tracer velocity and the dispersion coefficient as follows:

$$
\begin{aligned}
& D_{x x}=\alpha_{L}\left|V_{d y e}\right| \\
& D_{z z}=\alpha_{T}\left|V_{d y e}\right|
\end{aligned}
$$

where $\alpha_{L}$ is the longitudinal dispersivity and $\alpha_{T}$ is the transverse dispersivity.

\section{Results and discussion}

\subsection{Transport parameter estimates}

Longitudinal and transverse dispersion coefficients in Sand A and B are plotted in Figure 4 as a function of displacement of dye tracer. Note that longitudinal dispersion coefficient $D_{x x}$ describes a dispersion along a flow direction while transverse dispersion coefficient $D_{z z}$ is a lateral dispersion perpendicular to a flow direction. The left of the figure indicates the result for $0.129 \mathrm{~mm} / \mathrm{s}$ of rainfall intensity, which is approximately one half application rate shown in the right of the figure. Remarkably, dispersion coefficient has a tendency to decrease with the increase of displacement distance. A similar tendency shows in transverse dispersion coefficient estimates as seen in Figure 5. Lower application rate of rainfall exhibits a substantial decrease of both dispersion coefficients at a relatively shorter displacement distance. This is because greater flow velocity and water content induced from rainfall application directly affects the plume evolution associated with the value of the dispersion coefficient. Hence, increase of rainfall intensity results in considerable solute movement in a horizontal region as well as in faster travel time of solute from surface to water table.

Figures 6 and 7 show the results of longitudinal and transverse dispersivities, respectively. In contrast to the result of the dispersion coefficient, longitudinal dispersivity slightly increases with displacement distance and the degree of 

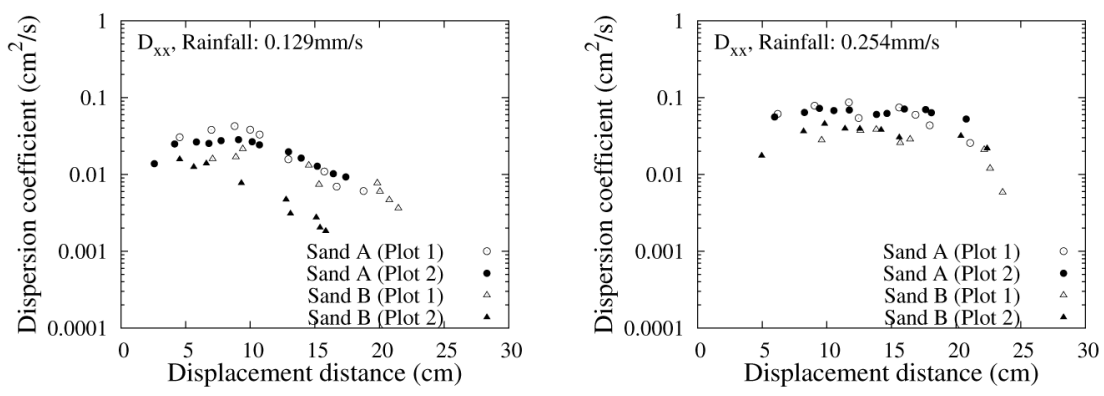

Figure 4: Longitudinal dispersion coefficient $D_{x x}$ estimates.
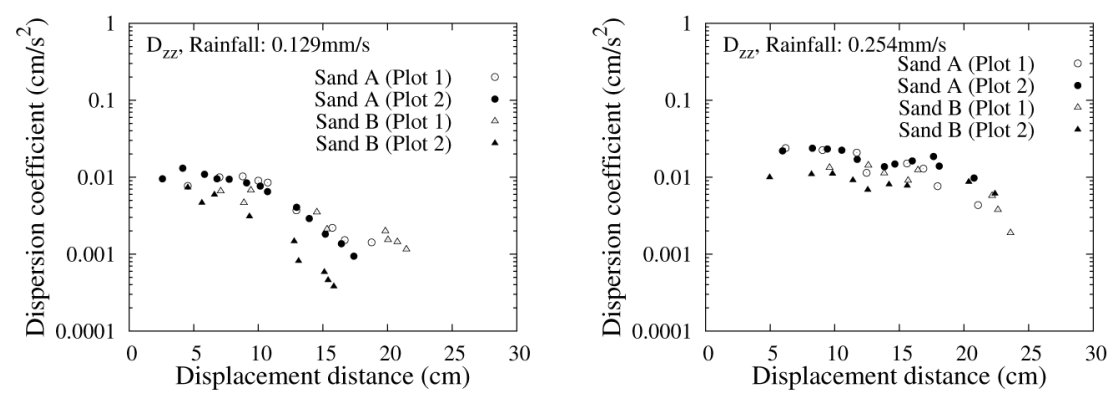

Figure 5: Transverse dispersion coefficient $D_{z z}$ estimates.

increase becomes larger as rainfall intensity decreases. It is inferred that longitudinal solute displacement depends largely on mixing of water and air whose distribution varies with the depth. The degree of increase of water content induced from rainfall application decreases as rainfall intensity is lower. Since diversity of solute movement pathway in porous media increases at lower saturation this process would make mobile region of water complicated and result in larger values for longitudinal dispersivity. On the other hand, transverse dispersivity remains constant regardless of rainfall intensity and displacement distance. This is probably because transverse dispersion in this flow field is a process under a relatively small variation of water content relative to longitudinal dispersion. Hence, transverse dispersion phenomenon under unsaturated conditions is clearly different from longitudinal dispersion phenomenon.

Figure 8 exhibits estimates of the dispersion coefficients as a function of tracer velocity with a relationship expressed in eqns (4) or (5). Under unsaturated condition, the dispersion coefficients increase as a linear function with increasing tracer velocity until this variable attains a certain value and start decreasing as tracer velocity increases beyond this value, leading to considerable scatter of the values. This may be due to the low mixing rate of water and air in this larger 

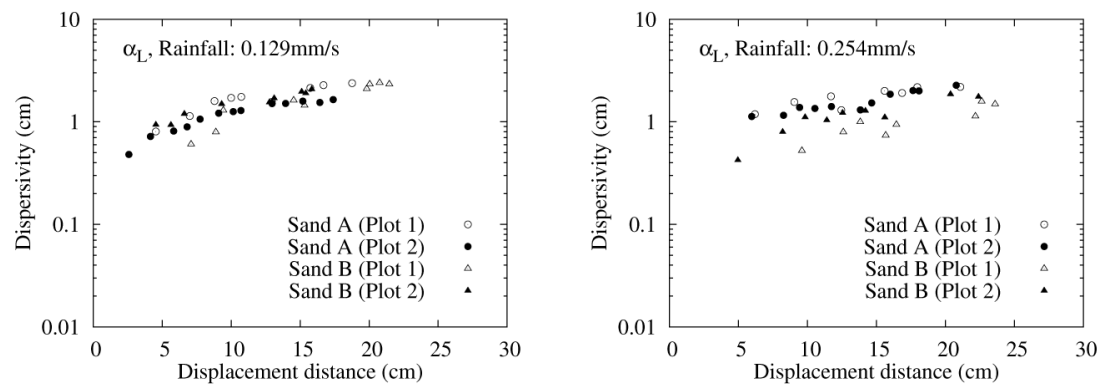

Figure 6: Longitudinal dispersivity $\alpha_{L}$ estimates.
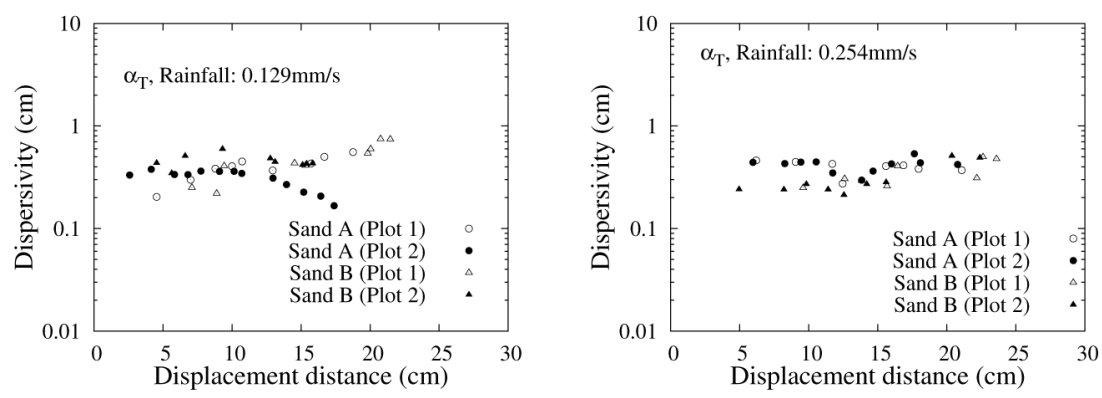

Figure 7: Transverse dispersivity $\alpha_{T}$ estimates.
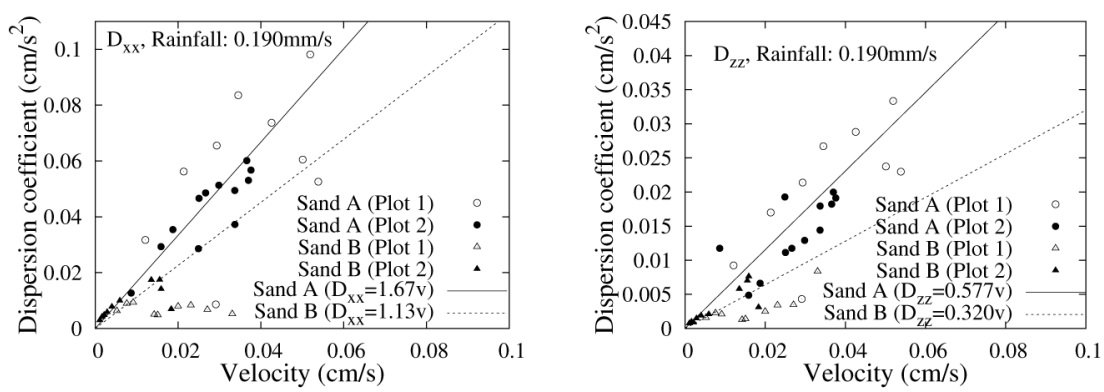

Figure 8: Relation between pore velocity and dispersion coefficients.

moisture content range. This finding is in accordance with the previous studies (e.g. de Smedt and Wierenga [7]; Matsubayashi et al. [8]), which are indicated that the dispersion coefficient is expected to be larger unsaturated conditions than under saturated conditions.

As a whole, estimates relevant to the transverse transport perpendicular to the flow direction becomes smaller than those of the longitudinal displacement 
Table 2: Comparison of dispersivity with column and flow tank experiment results.

\begin{tabular}{lc|cc}
\hline Condition & Material & $\alpha_{L}(\mathrm{~cm})$ & $\alpha_{T}(\mathrm{~cm})$ \\
\hline \hline Unsaturated $^{*}$ & Silica sand & $1.02^{`} 1.67$ & $0.320^{`} 0.577$ \\
Saturated [2] & Glass beads & $0.20^{`} 0.80$ & $0.051^{`} 0.12$ \\
Saturated [9] & Silica sand & $0.0017^{`} 0.18$ & $0.00070^{`} 0.029$ \\
Saturated [8] & Glass beads & $0.043^{`} 0.059$ & - \\
Saturated [10] & Glass beads & - & 0.013 \\
Unsaturated [8] & Glass beads & $0.25^{`} 2.8$ & - \\
Capillary fringe [2] & Glass beads & $0.060 ` 1.2$ & $0.0050^{`} 1.1$ \\
Unsaturated [7] & Glass beads & $0.41^{`} 4.33$ & - \\
Unsaturated [11] & Silt loam sand & $1.45^{`} 10.2$ & - \\
Unsaturated [12] & Sand & $10.0 ` 31.0$ & - \\
\hline * This study & \multicolumn{2}{|}{}
\end{tabular}

along with the flow direction for both dispersion coefficients and dispersivities, indicating that appropriateness of transport parameters estimated using image analysis.

\subsection{Comparison of column and flow tank experiment results}

Table 2 compares the results of the longitudinal and transverse dispersivities of this study with column and flow tank results reported in the literature conducted at approximately the same condition or in similar materials. The results obtained in this study are in good agreement with column test and flow tank test results under unsaturated flow condition, although the range of the longitudinal dispersivity is narrower than those in the literature. This is probably because the flow field in this study has relatively high water content and soil properties for both sand materials are similar each other. On the other hand, both dispersivities in unsaturated flow region are approximately one order larger than those in saturated flow region, indicating that the dispersion of solute can be related to water content where solute passes through. Image analysis is a useful tool for characterizing solute displacement and both longitudinal and transverse dispersion phenomena, even if parameter estimation is conducted with plume outline tracing.

\section{Conclusions}

In the present study, dye tracer experiments under unsaturated flow conditions have been conducted for homogeneous flow field filled with two different sizes of sands in order to investigate transport characteristics. Image analysis has applied 
for the estimation of dispersion coefficient and dispersivity in unsaturated porous media. These estimation values agree with experimental results in the literature, indicating the effectiveness of image analysis to parameter estimation problems. Experimental results revealed that transport parameters depend strongly on the water content where solute passes through.

\section{Acknowledgement}

The present study is supported, in part, by the Grant-in-Aid for Scientific Research, No. 16780169, the Ministry of Education, Culture, Sports, Science and Technology, Japan.

\section{References}

[1] Forrer, I., Kasteel, R., Flury, M. \& Flühler, H., Longitudinal and lateral dispersion in an unsaturated field soil. Water Resources Research, 35(10), pp. 3049-3060, 1999.

[2] Inoue, K., Inoue, K., Tanaka, T. \& Kobayashi, A., Image analysis to determine the dispersion coefficient and dispersivity in capillary and saturated zone. Proc. of the Int. Symp. on Advanced Experimental Unsaturated Soil Mechanics, eds. A. Tarantino, E. Romero \& Y. Cui, Balkema, pp. 467-473, 2005.

[3] Flury, M. \& Flühler, H., Tracer characteristics of Brilliant Blue FCF. Soil Science Society of America Journal, 59, pp. 22-27, 1995.

[4] Flury, M. \& Flühler, H., Brilliant Blue FCF as a dye tracer for solute transport studies - A toxicological overview. Journal of Environmental Quality, 23, pp. 1108-1112, 1994.

[5] van Genuchten M.Th., A closed-form equation for predicting the hydraulic conductivity of unsaturated soils. Soil Science Society of America Journal, 44(6), pp. 892-898, 1980.

[6] Bear, J., Dynamics of Fluids in Porous Media. Elsevier, 1972.

[7] de Smedt, F. \& Wierenga, P.J., Mass transfer in porous media with immobile water. Journal of Hydrology, 41, pp. 59-67, 1979.

[8] Matsubayashi, U., Devkota, L.P. \& Takagi, F., Characteristics of the dispersion coefficient in miscible displacement through a glass bead medium. Journal of Hydrology, 192, pp. 51-64, 1997.

[9] Inoue, K., Masaki, I., Setsune, N. \& Tanaka, T., Tracer experiments and parameter estimation in horizontally two-dimensional homogeneous porous media. Transactions of the Japanese Society of Irrigation, Drainage and Reclamation Engineering, 74(1), pp. 257-265, 2006.

[10] Robbins, G.A., Methods for determining transverse dispersion coefficient of porous media in laboratory column experiments. Water Resources Research, 25(6), pp. 1249-1258, 1989. 
[11] Bejat, L., Perfect, E., Quisenberry, V.L., Coyne, M.S. \& Haszler, G.R., Solute transport as related to soil structure in unsaturated intact soil blocks. Soil Science Society of America Journal, 64, pp. 818-826, 2000.

[12] Vanclooster, M., Mallants, D., Vanderborght, J., Diels, J., Van Orshoven, J. \& Feyen, J., Monitoring solute transport in a multi-layered sandy lysimeter using time domain reflectometry. Soil Science Society of America Journal, 59, pp. 337-344, 1995. 